\title{
A Stochastic Biometric Authentication Scheme using Uniformed GMM in Wireless Body Area Sensor Networks
}

\author{
Wei Wang, Department of Electrical Engineering and Computer Science, \\ South Dakota State University, USA \\ Kun Hua, Department of Electrical and Computer Engineering, \\ Lawrence Technological University, USA \\ Michael Hempel, Department of Computer and Electronics Engineering, \\ Dongming Peng, Department of Computer and Electronics Engineering, \\ Hamid Sharif, Department of Computer and Electronics Engineering, \\ University of Nebraska - Lincoln, USA \\ Hsiao-Hwa Chen, Department of Engineering Science, \\ National Cheng Kung University, Taiwan.
}

\begin{abstract}
In this paper, we propose an innovative low-cost biometric authentication scheme based on uniformed Gaussian mixture model (GMM) for secure communications within wireless body area sensor networks (WBASNs), to obviate the costprohibitive key exchange problem. In this approach, the sender Inter Pulse Interval (IPI) information is selected as the biometric key for authentication, and a statistical based signing scheme is developed using the uniformed GMM. Experimental analysis shows that the proposed approach effectively protects the communication channels in WBASN with a low computational complexity, significantly removing the need for secret key exchange and critical time synchronization overheads.
\end{abstract}

Index Terms-Wireless body area sensor network, biometric authentication, Gaussian mixture model.

\section{INTRODUCTION}

Recent advancements in wireless body area sensor networks (WBASNs) realize the evolution from a traditional desktop telemedicine platform to a wireless and mobile diagnosis system for delivering medical and healthcare services [1]. In a WBASN, wearable or implanted medical sensors around or inside the human body form a wirelessly interconnected

Paper submitted in 2010 IEEE PIMRC

Wei Wang (e-mail: wei.wangesdstate.edu) is with the Department of Electrical Engineering and Computer Science, South Dakota State University, USA.

Kun Hua (e-mail: khua@ltu.edu) is with Lawrence Technological University, USA.

Michael Hempel, Dongming Peng and Hamid Sharif (e-mail: \{mhempel, dpeng, hsharif\}@unlnotes.unl.edu) are with the Department of Computer and Electronics Engineering, University of Nebraska-Lincoln, USA.

Hsiao-Hwa Chen (e-mail: hshwchen @ieee.org) is with the Department of Engineering Science, National Cheng Kung University, Taiwan.

This work was supported in part by US NSF grant No. 0707944 for wireless sensor networks research and Taiwan National Science Council research gran NSC97-2219-E-006-004. network to collect an individual's medical information ubiquitously and to perform critical and complicated tasks collaboratively. For example, in a drug delivery system for diabetic assistance [2] as shown in Figure 1, glucose sensors inserted or implanted in the skin continuously monitor and wirelessly transmit the blood glucose levels to some microchip devices for accurate, timely, and efficient insulin release. In such a system, communication privacy among sensor nodes must be reliably protected since such applications involve sensitive medical information. Data integrity and authenticity must also be protected against modification of the wirelessly transmitted packets, because malicious and fraudulent actions may be extremely hazard (for example, alteration of drug dosages and treatment procedures) [1]. Other similar attacks include deliberation of health data leading to wrong diagnosis and treatment, as well as the falsification of alarms or suppression of real emergency alarms [3].

Limited researches have been reported in the literature regarding secure communications in WBASNs utilizing biometric information. Venkatasubramanian et al. [3] provided an overview of security solutions in pervasive healthcare systems, where biomedical information was advocated for securing data (collected by medical sensors) and for controlling access to health information managed by the pervasive healthcare systems. Cherukuri et al. [4] proposed a biometrics based key distribution scheme to secure the inter-sensor communications on the same human body. An identical pseudo-random number was generated through error correction code using biomedical properties of the human body recorded simultaneously at different locations, and this random number was used to encrypt, decrypt and securely distribute the symmetric key. Other similar researches regarding biometric security in WBASNs have been found in [9] [10] and [11]. However, all of the above research works were focused on secret key distribution issues, 


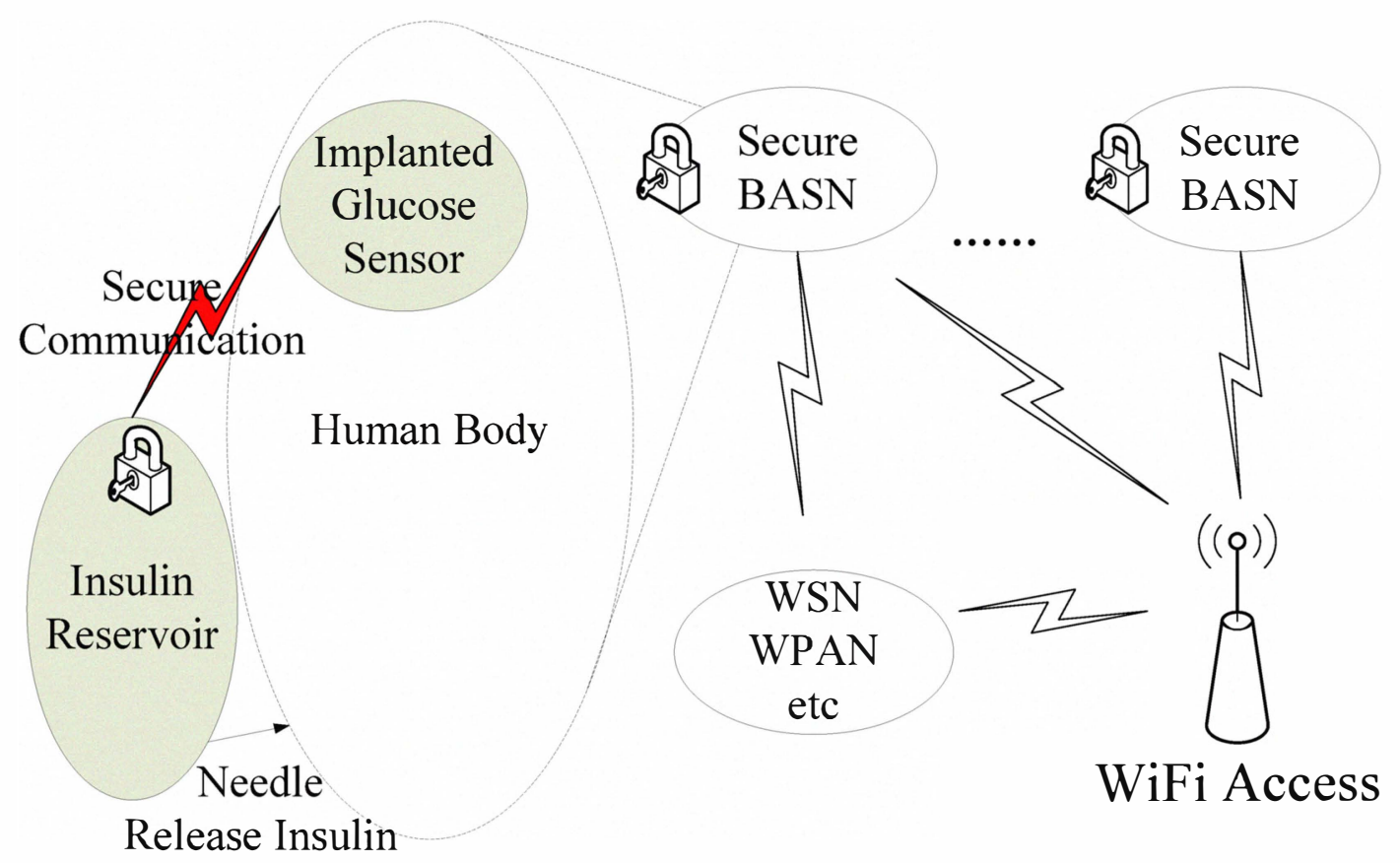

Fig. 1. An example of automatic drug delivery application in a WBASN for diabetic assistance.

while the specific challenges in biometric authentication, i.e., how to merge the payload data with the biometric information while preserving the statistical uniqueness of the biometric information was not fully discussed. Furthermore, all the aforementioned biometric based key exchange schemes need critical time synchronization because they need to record biometric information simultaneously at different locations of the same human body, which incurs considerable extra communication overheads in a extremely resource constrained WBASN. In this paper, we specifically consider the authentication challenges using biometric information, and propose a statistics based signing and verification scheme that eliminates the time synchronization overheads and other costs.

\section{BIOMETRIC AUTHENTICATION BASED ON GMM}

According to the recent researches reported in [1] [10] and [11], the timing information of heartbeats, i.e., Inter Pulse Interval (IPI), is an excellent candidate of biometric information which is desirable for cryptosystems in WBASNs. In this work, we use IPI signal as an example for securing inter-sensor communications within a WBASN. However, the proposed approach described in the later sections is general, and it should not be confined to IPI signals. Other biometric signals may also be easily and smoothly incorporated into the authentication scheme.
We can derive the likelihood of the GMM $\lambda$ representing the biometric authentication information $O$ in an $n$-point time period as follows

$$
p(O \mid \lambda)=\prod_{i=1}^{n} b\left(o_{i} \mid \lambda\right)=\prod_{i=1}^{n} \sum_{m=1}^{M} w_{m} \mathcal{N}_{m}\left(o_{i}\right)
$$

A log-based likelihood function can be used to evaluate the fitness of the modeling. Let $J(\lambda)$ denote this log-likelihood. It converts multiplication into a summation shown as follows

$$
J(\lambda)=\ln p(O \mid \lambda)=\sum_{i=1}^{n} \ln \sum_{m=1}^{M} w_{m} \mathcal{N}_{m}\left(o_{i}\right)
$$

To model the $n$-point biometric authentication information stored in $O$, we need to find the statistical characteristics $\lambda$ for a GMM achieving the maximum log-likelihood $J(\lambda)$. The calculation of the statistical characteristics $\lambda$ for biometric authentication can be effectively carried out by our proposed low cost signature signing process, which will be discussed in the next section.

\section{BIOMETRIC AUTHENTICATION ALGORITHM DEVELOPMENT}

\section{A. Signing and Verification Using Biometric IPI Signals}

In a secure drug delivery system for diabetic patient assistance, critical medical information such as glucose levels and 
drug delivery instructions compose the message data units (i.e., the payload denoted by $D$ ) to be transmitted wirelessly among the medical sensor nodes. To verify whether this message data unit is from the medical sensor node attached to the same body (i.e., from the trust zone), IPI signals (denoted by $I P I$ ) at the transmitter side are used to create the signature (denoted by $\{\lambda, J(\lambda)\}$ ), and attached to the message data unit. A hash function $H(I P I, D)$ is applied to the $I P I$, which is recorded for a period of time (forming a $n$-point discrete time series), and is also applied to the message data unit $D$, producing the transmitter side biometric authentication information $O$. The choice of the hash function may be generic and flexible. In this paper, we use XOR operation (or $O=I P I \oplus D$ ). The biometric authentication information $O$ is further modeled by our proposed uniformed GMM approach. The statistical characteristics $\lambda$ and the $\log$-likelihood $J(\lambda)$ of the biometric authentication information $O$ can therefore be extracted as the signature attached to the message data unit $D$.

During the signature verification process at the receiver's side, the $n$-point IPI signals recorded locally (denoted by $I P I^{\prime}$ ) are used to create the receiver side biometric authentication information $O^{\prime}$ with the received message data unit $D$ and the same hash function $H\left(I P I^{\prime}, D\right)$. GMM loglikelihood estimation is applied to the receiver side biometric authentication information $O^{\prime}$ and the signature $\{\lambda, J(\lambda)\}$ signed by the transmitter. If the IPI signals from both sides (i.e., $I P I$ and $I P I^{\prime}$ ) bear sufficient similarity, the receiver side $\log$-likelihood $J^{\prime}(\lambda)$ and transmitter side log-likelihood $J(\lambda)$ would be very close. If the difference of these log-likelihoods is over a threshold value $T$, the message data unit $D$ is regarded as false and discarded; otherwise it is authenticated and accepted as valid message data unit.

\section{B. Low Cost Signature Signing Process}

To acquire the optimal parameter values in the GMM representing biometric authentication information $O$, a uniformed Expectation - Maximization (EM) algorithm is proposed to obtain the maximum value of the log-likelihood function $J(\lambda)$. A new posterior probability notation $\beta_{j}\left(o_{i}\right)$ is defined in a way similar to that used in [5]-[8]. Intuitively, $\beta_{j}\left(o_{i}\right)$ denotes the probability that the $i$-th biometric authentication information point $o_{i}$ is generated by the $j$-th Gaussian mixture function. This posterior probability can be expressed as

$$
\beta_{j}\left(o_{i}\right)=\frac{w_{j} \mathcal{N}_{j}\left(o_{i}\right)}{\sum_{m=1}^{M} w_{m} \mathcal{N}_{m}\left(o_{i}\right)}
$$

To get the maximum value of $J(\lambda)$, it is necessary to have the first order derivative of mean and covariance of each Gaussian mixture to be zero, or

$$
\begin{gathered}
\frac{\partial J(\lambda)}{\partial \mu_{j}}=\sum_{i=1}^{n} \beta_{j}\left(o_{i}\right) \frac{o_{i}-\mu_{j}}{\sigma_{j}^{2}}=0 \\
\frac{\partial J(\lambda)}{\partial \sigma_{j}}=\sum_{i=1}^{n} \beta_{j}\left(o_{i}\right)\left[\frac{\left(o_{i}-\mu_{j}\right)^{T}\left(o_{i}-\mu_{j}\right)}{\sigma_{j}^{3}}-\frac{d}{\sigma_{j}}\right]=0
\end{gathered}
$$

Let $M$ be the number of Gaussian functions and $d$ be the dimension of samples. It is clear that there are $M \times(2+d)$ equations with $M \times(2+d)$ unknown parameters since the number of $w$ is $M$, the number of $\sigma^{2}$ is $M$, and the number of $\mu$ is $M \times d$. The calculation of these parameters in those equations can be effectively done by using our uniformed Expectation Maximization (EM) based iteration algorithm. It also guarantees a monotonically non-decreasing log-likelihood function. This uniformed EM for modeling the biometric authentication information is described in Algorithm 1.

\begin{tabular}{l}
\hline Algorithm 1 Uniformed EM algorithm for IPI Authentication. \\
\hline 1: Initialize IO and Read inputs. These IPI signals are \\
sampled into an $n$-point series and further processed by \\
de-noise filter. Then de-noised into input signals denoted \\
as IPI.
\end{tabular}

2: Create biometric authentication information $O=\left\{o_{1}, o_{2}, \ldots \ldots, o_{n}\right\}$ using transmitter side IPI samples. The information is calculated via the crypto hash function $O=I P I \oplus D$.

3: Initialize the first iteration with values $\lambda=$ $\left\{w_{j}, \mu_{j}, \sigma_{j}^{2} \mid j=1,2, \ldots \ldots . M\right\}$ according to the biometric measurement $O$. Default values of each weight are selected as $w_{j}=1 / M$, and $\mu_{j}$ is selected using a standard $k$-means algorithm. $\sigma_{j}^{2}$ can be calculated according to $\mu_{j}$ and the biometric authentication information $O$. Then, calculate $J(\lambda)$ using $\lambda$ and $O$.

4: Calculate $\beta_{j}\left(o_{i}\right)$ using the GMM parameters $\lambda$ and the biometric authentication information $O$. Calculate the new mean, covariance and weight values $\tilde{\mu}_{j}, \tilde{\sigma}_{j}^{2}, \tilde{w}_{j}$ using $\beta_{j}\left(o_{i}\right)$ and $O$. Calculate the new log-likelihood value $J(\tilde{\lambda})$ using the new GMM statistical characteristics $\tilde{\lambda}$ and $O$.

5: Let $\xi$ be a small positive value denoting the maximum relative uniform fitness. if $\left|\frac{J(\tilde{\lambda})-J(\lambda)}{J(\lambda)}\right| \leq \xi$, go to output step; else substitute the old GMM statistical characteristics values and uniformed log-likelihood values with the new model values: $\lambda \leftarrow \tilde{\lambda}, J(\lambda) \leftarrow J(\tilde{\lambda})$. Go back to step 4 for next iteration.

6: Output $\tilde{\lambda}$.

\section{EXPERIMENTS AND SIMULATIONS}

The IPI signals are acquired through a specifically designed algorithm. They are detected and measured by our wireless monitoring sensor implementation as exemplified in Figure 2. This platform is a part of the Wireless Infrastructure for Networks of Distributed Sensors (WINDS) research project at the University of Nebraska-Lincoln Peter Kiewit Institute in conjunction with University of Nebraska Medical Center (UNMC). 


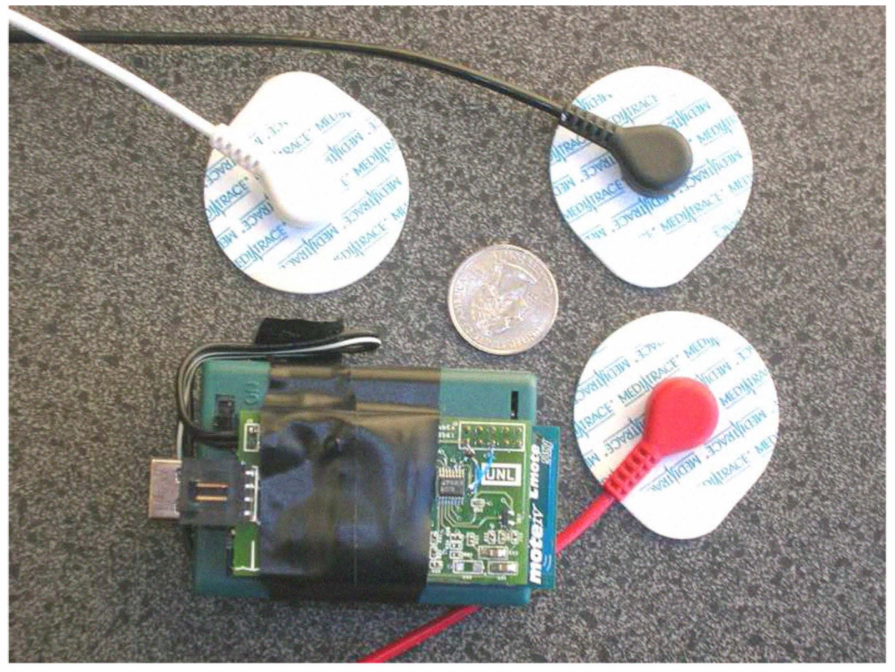

Fig. 2. The designed wireless IPI monitoring sensor connected to electrodes.

The performance of the proposed biometric authentication scheme is evaluated using biometric errors including False Rejection Rate (FRR), False Acceptance Rate (FAR) and Half Total Error Rate (HTER). Intuitively, the FRR denotes the event probability that a packet coming from the transmitting sensor node is falsely rejected by the receiving sensor node on the same human body; and the FAR denotes the event probability that a packet coming from the sensor node on a different human body is erroneously accepted by the receiving sensor node on another human body. The HTER can be determined with FRR and FAR by

$$
H T E R=\frac{1}{2}(F R R+F A R)
$$

To acquire FRR, FAR and HTER evaluation metrics, we have performed extensive experiments, and multiple sessions of 30 minutes IPI data are acquired for each subject. Each data session is used to create the biometric authentication information $O$ modeled by GMM, and the likelihood of which is cross compared with the biometric measurements from all the other sessions. Multiple GMM modeling and likelihood comparison processes are performed to acquire each average FRR, FAR and HTER point.

Figure 3 shows the authentication performance in terms of FRR, FAR and HTER for different decision thresholds $T$. The decision threshold $T$ denotes how stringent the authentication scheme is - a smaller value of the decision thresholds requires higher similarities between the sender side and receiver side IPI signals, and vice versa. When the decision threshold values are low, FRR dominates the decision errors because more packets from sensors on the same human body may be falsely rejected by those receiver sensors due to the slight differences among the IPI signals on different parts of the same human body. This is especially prominent for $T=0$ case in this figure, where any small difference between sender and receiver IPI signals incurs a decision error. When the decision threshold increases, FRR decreases and FAR increases accordingly. If the decision threshold is large, FAR dominates the decision errors and any packet from a sensor on a different human body will be erroneously accepted by the receiver sensor due to the high tolerance of IPI signal dissimilarities.

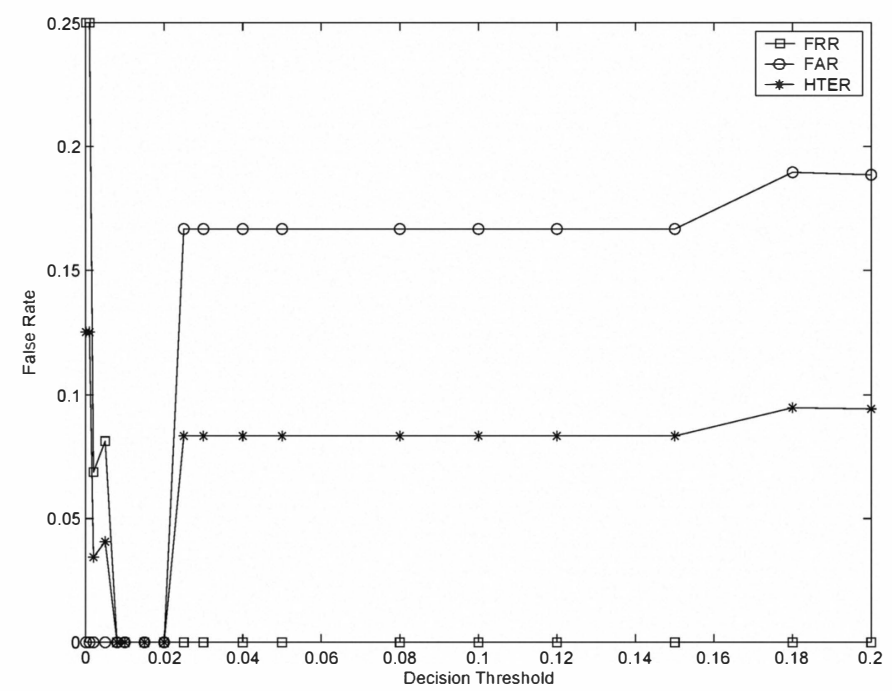

Fig. 3. FRR, FAR and HTER performances for different decision thresholds. In this experiment, the number of samples $n$ is 60 and the number of Gaussian mixtures $M$ is two.

\section{CONCLUSION}

The biometric information carried by the blood circulation system in a human body forms a secure communication medium which is uniquely available for WBASNs. In this study, we have proposed a biometric based authentication scheme to secure the inter-communications among medical sensors in a WBASN only utilizing the locally captured IPI signals, while eliminating the key exchange problems. In the proposed scheme, a statistical based signing process has been proposed to resolve the time synchronization challenges. Experimental works have shown that the proposed authentication scheme effectively secures the communications among sensors on the same human body.

\section{FUTURE WORKS}

The potentials for improving the proposed biometric authentication scheme in WBASNs reside in two aspects: 1) the design of a more appropriate crypto hush function, and 2) the selection of more appropriate biometric features in addition to IPI signals. As described in Section III, the XOR based hash function is applied to the IPI signals and message data unit. Since the proposed signing and signature verification processes both are independent of the crypto hash function, other hash functions that preserve the statistical characteristics of the biometric signals (e.g., IPI signals) are also desirable candidates for such authentication schemes. Furthermore, the proposed signature signing and verification processes described in Section III are independent of the biometric information selection. In this study, although IPI is selected as the biometric feature for authentication, where 
other biometric factors such as the temporal dynamics of IPI, HRV or the combinations of such signals can also be applied seamlessly to the proposed scheme. Further investigations of these open issues may contribute to the studies on tackling the formidable yet meaningful security challenges in extremely resource constrained WBASNs.

\section{REFERENCES}

[1] C. Poon, Y. Zhang, S. Bao, "A novel biometrics method to secure wireless body area sensor networks for telemedicine and m-health," IEEE Commun. Mag,pp.73-81, Apr. 2006.

[2] http://www.diabetesmine.com/diabetes_product_parade/index.html

[3] K. Venkatasubramanian, S. Gupta, Chapter 15 Security Solutions for Pervasive Healthcare, Security in distributed, grid, mobile, and pervasive computing, Auerbach Publications, CRC Press, pp 443-464, 2007.

[4] S. Cherukuri, K. Venkatasubramanian, S. Gupta, "Biosec: a biometric based approach for securing communication in wireless networks of biosensors implanted in the human body," in Proc. IEEE Int. Conf. Parallel Processing Workshops,pp.432-439, Oct. 2003.

[5] J.Jang, Data Clustering and Pattern Recognition . chap.6, Gaussian Mixture Model.

[6] M.Shi, A.Bermak, "An efficient digital VLSI implementation of Gaussian mixture models-based classifier," IEEE Trans. VLSI syst., vol.14, no.9, pp.962-974, Sept.2006.

[7] J.Gauvain, C.Lee, "Maximum a posteriori estimation for multivariate Gaussian mixture observations of Markov chains," IEEE Trans. Speech and Audio Process., vol.2, no.2, pp.291-298, Apr.1994.

[8] D.Reynolds, T.Quatieri, R.Dunn, "Speaker verification using adapted Gaussian mixture models," Dig. Signal Process.,vol.10,no.1-3,2000.

[9] A. Juels, M. Wattenberg, "A fuzzy commitment scheme," in Proc. $6^{\text {th }}$ ACM Conf. Comp. and Commun. Sec.,pp.28-36, Nov. 1999.

[10] S. Bao, Y. Zhang, L. Shen, "Physiological signal based entity authentication for body area sensor networks ad mobile healthcare systems," in Proc. IEEE Engineering in Medicine and Biology,pp.2455-2458, Sept. 2005.

[11] S. Bao, Y. Zhang, "A design proposal of security architecture for medical body sensor networks," in Proc. IEEE International Workshop on Wearable and Implantable Body Sensor Networks,4pp. Apr. 2006. 